\title{
Venous occlusion plethysmography vs. Doppler ultrasound in the assessment of leg blood flow kinetics during different intensities of calf exercise
}

Elaine Murphy, Joel Rocha, Norita Gildea, Simon Green and Mikel Egaña

This is a post-peer-review, pre-copyedit version of an article published in European Journal of Applied Physiology. The final authenticated version is available online at: http://dx.doi.org/10.1007/s00421-017-3765-z 


\section{TITLE}

Venous occlusion plethysmography vs. Doppler ultrasound in the assessment of leg blood flow kinetics during different intensities of calf exercise

\section{AUTHORS}

Elaine Murphy ${ }^{1}$, Joel Rocha ${ }^{2}$, Norita Gildea ${ }^{1}$, Simon Green $^{3}$, Mikel Egaña $^{1}$

\section{AFFILIATIONS \& ADDRESSES}

1. School of Medicine, Department of Physiology, Trinity College Dublin, Dublin 2, Ireland.

2. Division of Sport and Exercise Sciences, Abertay University, UK

3. School of Science and Health, Western Sydney University, Australia.

\section{CONTACT INFORMATION (corresponding author):}

Mikel Egaña

Department of Physiology,

Trinity College Dublin,

Dublin 2, Ireland.

E-mail: megana@tcd.ie

Telephone: +35318961770

Fax: +35316793545 


\begin{abstract}
:
Purpose It has recently been shown that venous occlusion plethysmography (VOP) can successfully assess the rate of increase in leg blood flow (LBF) (LBF kinetics) responses during calf exercise, but there is lack of data supporting its validity. Methods Using Doppler ultrasound (DU) as a criterion standard technique, we tested the hypothesis that VOP would provide similar estimates of LBF kinetics responses as DU during calf plantar-flexion exercise at a range of different intensities. Ten healthy men performed repeated intermittent calf plantar-flexion contractions $(3$ s duty cycles, 1 s contraction / 2 s relaxation) at 30, 50 and 70\% maximum voluntary contraction (MVC) on different days. Results Resting LBF values were significantly $(P<0.05)$ larger for DU than VOP but the overall mean LBF responses during exercise were not different $(P>0.05)$ between DU and VOP (30\% MVC: $330 \pm 78$ vs. $313 \pm 92$ $\mathrm{ml} / \mathrm{min} ; 50 \%$ MVC: $515 \pm 145$ vs. $483 \pm 164 \mathrm{ml} / \mathrm{min} ; 70 \%$ MVC: $733 \pm 218$ vs. $616 \pm 229 \mathrm{ml} / \mathrm{min})$. LBF kinetics analyses revealed that the end-amplitude at the highest intensity (70\% MVC) was significantly higher when measured by DU compared with VOP, but all other kinetics parameters were not different between VOP and DU. Conclusions Given that these slight differences in amplitude observed during exercise can be explained by differences in vascular regions which the two techniques assess, our results suggest that VOP can accurately assess LBF kinetics responses during calf plantar-flexion exercise at intensities between 30 and 70\% MVC.
\end{abstract}

\title{
Keywords:
}

Blood flow, exercise, venous occlusion plethysmography, Doppler ultrasound, validity

\section{Abbreviations:}

A: Amplitude.

ANOVA: Analysis of variance

CV: Coefficient of variation.

DBP: Diastolic blood pressure.

DU: Doppler ultrasound.

ECG: Electrocardiogram.

ICC: Intraclass correlation coefficient.

LBF: Leg blood flow.

MAP: Mean arterial blood pressure.

MVC: Maximal voluntary contraction. 
PBV: Popliteal blood velocity.

PDiam: Popliteal arterial diameter.

SBP: Systolic blood pressure.

SD: Standard deviation

TD: Time delay.

VOP: Venous occlusion plethysmography. 


\section{Introduction}

The rate at which leg muscle blood flow (LBF) is increased at the onset of exercise (LBF kinetics) has been shown to be closely related to the rate of increase in muscle/pulmonary oxygen uptake at exercise onset (i.e. $\dot{\mathrm{VO}}_{2}$ kinetics) (MacDonald et al. 1998; duManoir et al. 2010). Given that blunted or slowed $\dot{\mathrm{V}} \mathrm{O}_{2}$ kinetics responses are related to earlier onset of fatigue and reduced exercise tolerance, which is related to morbidity and mortality in all ages (Paterson et al. 2007), it is paramount to accurately quantify and assess the initial increase in the LBF response during exercise. This is particularly relevant for patients with peripheral vascular disease whereby information about the leg hyperaemic response during exercise is of clinical importance in the diagnosis and monitoring of those patients (Green 2002).

Various techniques have been employed to quantify this hyperaemic response to exercise (Radegran 1999) including magnetic resonance imaging (Anderson et al. 2009) and position emission tomography (Heinonen et al. 2007). The most common techniques, often considered as criterion standards, are thermodilution and Doppler ultrasound (DU) (Andersen and Saltin 1985; Gonzalez-Alonso et al. 2006; Koskolou et al. 1997; Radegran and Saltin 1998; Saunders et al. 2005). While these two techniques provide similar estimates of leg blood flow during exercise (Radegran 1997), thermodilution is invasive and has poor temporal resolution for the measurement of the hyperaemic response (Saunders et al. 2005). DU permits continuous measurements on a beat-by-beat basis due to its higher temporal resolution allowing blood flow measurements during exercise; however, it is restricted to single conduit arteries, and thus, it is not suited to assess arterial inflow into limbs that present arterial collateralization due to vascular disease.

Venous occlusion plethysmography (VOP) is a common non-invasive, blood flow measurement technique that measures whole limb blood flow by assessing the changes in limb circumference and is therefore, a particularly useful method for patients with diseases affecting vascularisation of the lower limb. VOP is a well established technique in the study of vascular control and adaptation that is generally used under resting or immediately after exercise conditions given that the method requires that the limb is immobilized during the period of assessment (Joyner et al. 2001). Under these conditions conflicting evidence exists regarding the accuracy of this technique and the correlation between VOP and other blood flow measurement techniques, including DU, radioisotope washout or dye-dilution methods (Adiseshiah et al. 1984; Bystrom et al. 1998; Saltin 2007; Bernink et al. 1982; Hiatt et al. 1989; Jorfeldt and Rutberg 1990; Lubbers et al. 1979; Pallares et al. 1994; Tschakovsky et al. 1995; Radegran 1999). 
However, Green et al. (2011) recently reported similar estimates of LBF responses during calf exercise between VOP and DU during relaxation periods between intermittent contractions performed across a wide range of intensities (Green et al. 2011). In addition, confirming early findings by Barcroft and Millen (Barcroft and Millen 1939), Green et al. (2011) showed that LBF responses during contractions at different intensities were not greater than LBF values at rest demonstrating that the hyperaemic response is limited during the contraction and it manifests mainly during relaxation (Green et al. 2011).

We have recently shown that VOP can successfully be used to assess LBF kinetics responses during calf exercise in healthy participants (Egaña and Green 2005; Reeder and Green 2012) as well as in participants with type 2 diabetes (MacAnaney et al. 2011; Kiely et al. 2014); but there is lack of data supporting the validity of VOP in measurements of limb blood flow kinetics. Accordingly, the aim of the present study was to investigate the accuracy of VOP as a measurement of leg blood flow kinetics during calf plantar-flexion exercise at different exercise intensities using simultaneous DU measurements as a criterion standard technique. We hypothesised that VOP would provide similar estimates of LBF kinetics responses to exercise as DU.

\section{Materials and Methods}

\section{Participants}

Ten young men (mean \pm SD; age: $28 \pm 4$ yr; height: $180 \pm 9 \mathrm{~cm}$; body mass: $80 \pm 11 \mathrm{~kg}$, body mass index: $25.1 \pm$ $2.2 \mathrm{~kg} \cdot \mathrm{m}^{-2}$; leg volume: $3.38 \pm 0.56 \mathrm{~L}$ ) volunteered to take part in this study. All participants were non-smokers with no apparent cardiovascular disease (assessed by medical questionnaire and physical examination as well as anklebrachial index measurement) and regularly undertook moderate to vigorous exercise at least 2-3 times per week. To avoid diurnal fluctuations in fatigue and overall exercise performance, all experimental sessions were held at the same time of day and participants were required to refrain from heavy exercise and caffeine or alcohol consumption for $24 \mathrm{~h}$ before each visit to the laboratory. Each participant gave written informed consent to participate in this study, which was conducted according to the Declaration of Helsinki and approved by the Faculty of Health Science Research Ethics Committee, Trinity College Dublin.

\section{Experimental design}

All participants visited the cardiovascular performance laboratory at Trinity College Dublin on two occasions separated by at least 48 hours. On visit one, participants were initially familiarised with the calf plantar-flexion 
exercise protocol and performed 3 maximal voluntary contractions (MVC) in the prone position separated by one minute from which the highest effort was taken as MVC. Participants rested for $\sim 20$ min and then performed five intermittent plantar-flexion exercise bouts of $6 \mathrm{~min}$. The first two bouts were performed at $30 \% \mathrm{MVC}$, the $3^{\text {rd }}$ and $4^{\text {th }}$ bouts at $50 \%$ MVC and the last bout at 70\% MVC. Each exercise bout was separated by a resting period of 10 min. On visit two, participants repeated the same five exercise bouts following the same order and resting periods as per visit one. Testing was conducted in a quiet, darkened room where room temperature was kept constant at $\sim 22^{\circ} \mathrm{C}$.

\section{Calf exercise}

Intermittent ( $3 \mathrm{~s}$ duty cycle: $1 \mathrm{~s}$ contraction / $2 \mathrm{~s}$ relaxation) isometric plantar-flexion exercise was performed with the right and dominant leg while using a custom-built calf ergometer as previously described (Egaña and Green 2007, 2005). The set-up of the ergometer was slightly modified so that participants in the present study were in the prone position (instead of the supine position used in our previous studies). The prone position was used to better identify the popliteal artery for the DU measurements. All measurements were recorded in the horizontal position to remove the effects of hydrostatic pressure and to minimise cardiopulmonary baroreceptor-mediated changes in muscle sympathetic nerve activity (Ray et al. 1993). Briefly, the participant was secured to the ergometer with a harness that minimised the backward displacement of the body during each contraction while hand gripping was not allowed. The participant's foot was attached to an immobile footplate, which is connected to its own strain gauge and against which force can be applied when the participant plantar-flexes the foot. The resultant force was amplified and sampled at $40 \mathrm{~Hz}$ before being processed by a PowerLab analog-to-digital converter (ML 795, AD Instruments) and displayed on a screen visible to the participant (Chart v6.0, AD Instruments). Participants used this visual display to control their effort and to conform to specific instructions given by the investigator.

\section{Leg blood flow}

LBF was assessed at rest (only during the $60 \mathrm{~s}$ period preceding the first exercise bout at $30 \% \mathrm{MVC}$ ) and during exercise (i.e. during the relaxation periods between intermittent contractions) using VOP and DU simultaneously (see immediately below). LBF measurements were performed by the same operator on both visits.

VOP and leg blood flow: Briefly, a mercury-in-silastic strain gauge (Hokanson EC-6, USA) was placed around the widest part of the subject's right calf and connected to the plethysmograph (Hokanson EC-6, USA). A cuff 
(Hokanson, USA) placed around the upper right thigh of the subject was inflated to a constant pressure of $\sim 55$ $\mathrm{mmHg}$ for the duration of the exercise. The pressure of $\sim 55 \mathrm{mmHg}$ was chosen to occlude venous return without interfering with arterial BF (Egaña and Green 2005; Hunt et al. 2016). LBF during exercise was assessed by measuring the change in leg volume detected by the strain gauge over the $2 \mathrm{~s}$ relaxation period between contractions (Egaña and Green 2005). To record resting LBF values, the cuff was inflated to $\sim 55 \mathrm{mmHg}$ at $10 \mathrm{~s}$ intervals for 1 min while the participant rested. The plethysmographic estimates of LBF, expressed relative to the resting limb volume $\left(\mathrm{ml} \cdot 100 \mathrm{ml}^{-1} \cdot \mathrm{min}^{-1}\right)$ were converted to millilitres per minute $(\mathrm{ml} / \mathrm{min})$ using an estimate of each participant's leg volume obtained from the water displacement method of the leg (Kaulesar Sukul et al. 1993).

DU and leg blood flow: Doppler ultrasound imaging was performed using a Zonare Z.One Ultra apparatus operated in pulsed wave mode (3.5 MHz) with a flat L8-3 transducer, operating at a frequency of $7 \mathrm{MHz}$. Ultrasound images were triggered on the R-wave pulse of an electrocardiogram (ECG) signal captured using a video capture card (Matrox Orion HD, Matrox, Quebec, Canada) and recorded with a vascular imaging software (Vascular Imager HD: 6.3.3, IO, USA). The gate width was set to $11 \mathrm{~mm}$ for all participants and all blood flow measurements were insonated at a $60^{\circ}$ angle to standardise measurements (Radegran 1999). To determine transducer placement, the popliteal artery was located using 2D ultrasound with an L3 transducer operating in B-mode. The transducer was manipulated to establish correct alignment along its longitudinal axis and to ensure full isolation of the artery lumen without contamination from the venous signal. Transducer position was recorded by directly marking the skin to assist repositioning of the probe during the test and on subsequent tests. During the test, the investigator manipulated the transducer to preserve the best Doppler signal directed by auditory and visual feedback. Popliteal artery mean blood velocity (PBV) and luminal diameter (PDiam, i.e. end-diastolic diameter) were recorded at each cardiac cycle and analyzed for each duty cycle (i.e. averaged over the $2 \mathrm{~s}$ relaxation period) with a custom-designed edge detection and wall tracking software (Medical Imaging Applications, Vascular Research Tools 5, IO, USA). The popliteal blood velocity $(\mathrm{PBV})\left(\mathrm{cm} \cdot \mathrm{s}^{-1}\right)$ and the PDiam (radius in $\mathrm{cm}$ ) were used to calculate LBF (ml/min) using DU, as LBF $=\mathrm{PBV} \pi r^{2} 60$, where $r$ is the vessel radius. Resting LBF values were recorded at $10 \mathrm{~s}$ intervals for 1 min and then averaged.

Preliminary testing revealed that LBF flow responses using both techniques were not different between the two days of testing, and were repeatable as demonstrated by moderate variability, good agreement and consistency. The coefficient of variation for LBF at rest and between $\min 4$ and 6 of the constant load bouts (i.e. steady-state 
condition) was between 25 and $28 \%$ for DU and between 30 and 35\% for VOP, with intraclass correlation coefficients (ICC) between $0.70-0.86$ for DU and 0.60-0.91 for VOP.

\section{Data Analysis}

LBF responses estimated by DU and VOP for the exercise bouts at each intensity from both exercise days were averaged to yield a temporal profile of LBF for each subject. It was evident that in addition to the two growth phases commonly observed in the literature during low to moderate intensity hyperaemia (Radegran and Saltin 1998; Saunders et al. 2005), a fast decay phase followed the first growth phase in most responses, as recently described (Kiely et al. 2014; Reeder and Green 2012). In addition, a second slow decay (after the second growth phase) was also evident in many participants $(30 \% \mathrm{MVC}, n=6 ; 50 \% \mathrm{MVC}, n=7 ; 70 \% \mathrm{MVC}, n=3)$. Therefore, to better represent the physiological responses and to enable a more accurate estimate of the parameters defining the first three phases, a quadraphasic model was fitted in participants in whom a second decay was apparent, but the second decay was not compared across measurement techniques. The following two empirical models represented by their corresponding functions (see below) were fitted to the individual time series of LBF:

Triphasic (two growth and one decay phases) model:

$y(\mathrm{t})=\mathrm{A}_{0}+\mathrm{A}_{1}\left(1-e^{(-(\mathrm{t}-\mathrm{TD} 1) / \tau 1)}\right) \mathrm{F} 1-\mathrm{A}_{2}\left(1-e^{(-(\mathrm{t}-\mathrm{TD} 2) / \tau 2}\right) \mathrm{F} 2+\mathrm{A}_{3}\left(1-e^{(-(\mathrm{t}-\mathrm{TD} 3) / \tau 3)}\right) \mathrm{F} 3$

Quadraphasic (two growth and two decay phases) model:

$y(\mathrm{t})=\mathrm{A}_{0}+\mathrm{A}_{1}\left(1-e^{(-(\mathrm{t}-\mathrm{TD} 1) / \tau 1)}\right) \mathrm{F} 1-\mathrm{A}_{2}\left(1-e^{(-(\mathrm{t}-\mathrm{TD} 2) / \tau 2}\right) \mathrm{F} 2+\mathrm{A}_{3}\left(1-e^{(-(\mathrm{t}-\mathrm{TD} 3) / \tau 3)}\right) \mathrm{F} 3-\mathrm{A}_{4}\left(1-e^{(-(\mathrm{t}-\mathrm{TD} 4) / \tau 4)}\right) \mathrm{F} 4$

where

$$
\begin{aligned}
& F 1=0 \text { for } \mathrm{t}<\mathrm{TD} 1 \text { and } \mathrm{F} 1=1 \text { for } \mathrm{t} \geq \mathrm{TD} 1 \\
& \mathrm{~F} 2=0 \text { for } \mathrm{t}<\mathrm{TD} 2 \text { and } \mathrm{F} 2=1 \text { for } \mathrm{t} \geq \mathrm{TD} 2 \\
& \mathrm{~F} 3=0 \text { for } \mathrm{t}<\mathrm{TD} 3 \text { and } \mathrm{F} 3=1 \text { for } \mathrm{t} \geq \mathrm{TD} 3 \\
& \mathrm{~F} 4=0 \text { for } \mathrm{t}<\mathrm{TD} 4 \text { and } \mathrm{F} 4=1 \text { for } \mathrm{t} \geq \mathrm{TD} 4
\end{aligned}
$$

$y(\mathrm{t})$ is LBF at time $t, \mathrm{~A}_{0}$ is the baseline amplitude, $\mathrm{A}_{1}, \mathrm{~A}_{2}, \mathrm{~A}_{3}$, and $\mathrm{A}_{4}$ are the amplitudes, $\mathrm{TD}_{1}, \mathrm{TD}_{2}, \mathrm{TD}_{3}$, and $\mathrm{TD}_{4}$ are the time delays and $\tau_{1}, \tau_{2}, \tau_{3}$, and $\tau_{4}$ are the time constants of the first (rapid growth), second (rapid decay), third (slow growth), and fourth (slow decay) phases respectively. The parameters F1 - F4 are conditional expressions that limit the fitting of a particular phase to the period at and beyond the time delay associated with that phase. The two models were fitted to LBF data using a weighted least-squares nonlinear regression procedure and the 
Marquardt-Levenberg algorithm (TableCurve 2D, Jandel Scientific, USA). For all models, data that exceeded the 95\% prediction intervals during an initial fit of a model were excluded. The steady-state amplitude, referred to as End A, for the two models was calculated as follows:

Triphasic model:

End $\mathrm{A}=\mathrm{A}_{0}+\mathrm{A}_{1}\left(1-e^{-(360-\mathrm{TD} 1) / \tau 1)}\right)-\mathrm{A}_{2}\left(1-e^{-(360-\mathrm{TD} 2) / \tau 2)}\right)+\mathrm{A}_{3}\left(1-e^{-(360-\mathrm{TD} 3) / \tau 3)}\right)$

Quadraphasic model:

End $\mathrm{A}=\mathrm{A}_{0}+\mathrm{A}_{1}\left(1-e^{-(360-\mathrm{TD} 1 / \tau 1)}\right)-\mathrm{A}_{2}\left(1-e^{-(360-\mathrm{TD} 2 / \tau 2)}\right)+\mathrm{A}_{3}\left(1-e^{-(360-\mathrm{TD} 3 / \tau 3)}\right)-\mathrm{A}_{4}\left(1-e^{-(360-\mathrm{TD} 4 / \tau 4)}\right)$

\section{Statistical Analyses}

Estimated parameters of the LBF kinetics responses as well as mean LBF responses between VOP and DU at each exercise intensity (30, 50 and 70\% MVC) were compared using a two-way repeated measures ANOVA and Tukey's post-hoc test. Similarly, PDiam values at rest, end $A_{1}$ and end exercise (using DU) at each exercise intensity were compared using a two-way repeated measures ANOVA and Tukey's post-hoc test. Mean PDiam values at each of the three intensities were compared using the non-parametric Friedman test and Student-Newman Keuls test. To test the consistency of LBF measurements between the two days of testing coefficient of variation $(\mathrm{CV}=\mathrm{standard}$ deviation/mean*100) and ICC were performed whereas to test the level of agreement between the two days of testing as well as between the two measurement techniques Bland-Altman plots were constructed. All statistical analyses were performed using SigmaPlot (v12; Systat Software, CA, USA) with the level of significance set to $P$ $<0.05$. All values are expressed as mean $\pm \mathrm{SD}$ (parametric data) or median and interquartile ranges (non-parametric data).

\section{Results}

Mean estimates of leg blood flow using DU and VOP

The mean LBF responses obtained during the relaxation phases in each duty cycle (every $3 \mathrm{~s}$ ) employing both VOP and DU are presented in Fig. 1. Mean resting LBF values were significantly $(P<0.05)$ larger for DU $(172 \pm 47$ $\mathrm{ml} / \mathrm{min})$ compared with VOP $(73 \pm 35 \mathrm{ml} / \mathrm{min})$, but the overall mean LBF responses averaged over the 6 min bouts at the 3 exercise intensities were not different between the two techniques (30\% MVC, DU: $330 \pm 78 \mathrm{ml} / \mathrm{min}, \mathrm{VOP}$ : $313 \pm 92 \mathrm{ml} / \mathrm{min} ; 50 \%$ MVC: DU: $515 \pm 145 \mathrm{ml} / \mathrm{min}, \mathrm{VOP}: 483 \pm 164 \mathrm{ml} / \mathrm{min} ; 70 \%$ MVC: DU: $733 \pm 218 \mathrm{ml} / \mathrm{min}$ 
VOP: $616 \pm 229 \mathrm{ml} / \mathrm{min})$.

\section{Mean estimates of popliteal artery diameter and blood velocity using $D U$}

The mean PDiam and PBV during the relaxation phases in each duty cycle (every $3 \mathrm{~s}$ ) employing DU are presented in Fig. 3. The overall PDiam averaged over the 6 min bouts at the 3 intensities were significantly $(P<0.05)$ larger at $70 \%(5.52[5.47-5.58])$ and $50 \%(5.40[5.38-5.42])$ compared with $30 \%$ MVC (5.30 [5.21 - 5.35]) and were also significantly larger $(P<0.05)$ at $70 \%$ compared with 50\% MVC intensities. PDiam at the end of exercise were significantly $(P<0.05)$ larger than resting PDiam, and PDiam at the end of $\mathrm{A}_{1}$ tended to be larger than resting PDiam ( $\mathrm{P}=0.09)$ within each of the three intensities (main effect time, $P<0.05$ ), but were not different among intensities (30\% MVC, rest: $5.26 \pm 0.54 \mathrm{~mm}, \mathrm{~A}_{1}: 5.28 \pm 0.41 \mathrm{~mm}$, end exercise: $5.37 \pm 0.51 \mathrm{~mm}$; $50 \%$ MVC: rest: $5.23 \pm 0.48 \mathrm{~mm}, \mathrm{~A}_{1}: 5.37 \pm 0.45 \mathrm{~mm}$, end exercise: $5.37 \pm 0.50 \mathrm{~mm} ; 70 \%$ MVC: rest: $5.23 \pm 0.48 \mathrm{~mm}, \mathrm{~A}_{1}: 5.50 \pm$ $0.66 \mathrm{~mm}$, end exercise: $5.54 \pm 0.64 \mathrm{~mm})$.

\section{Leg blood flow kinetics responses using DU and VOP}

LBF kinetics responses obtained using VOP and DU during plantar-flexion exercise at 30\%, 50\% and $70 \% \mathrm{MVC}$ are presented in Table 1 and individual plots from a representative individual are presented in Fig. 2 In addition, Bland Altman plots for $\mathrm{A}_{1}$ and End A parameters for all three intensities between the two measurement techniques are shown in Fig 4. DU estimates of resting blood flow were significantly larger than those measured with VOP $(P$ $<0.05)$. The end amplitude (End A) at 70\% MVC was also significantly larger $(P<0.05)$ for DU than VOP. No other parameters of the LBF kinetics responses were different between DU and VOP.

\section{Discussion}

To our knowledge this is the first time that leg blood flow kinetics during exercise at a range of different intensities were compared between VOP and DU. In agreement with our hypothesis, the current study provides evidence that VOP is an acceptable measurement technique for the assessment of the dynamic response characteristics of leg blood flow during calf plantar-flexion exercise. Nevertheless, resting LBF values and end-exercise responses at the highest intensity were larger when using DU.

\section{LBF kinetics during exercise}

Immediately at the onset of exercise, the rate of metabolic processes increases significantly. By identifying the 
discrete phases of the dynamic response of blood flow to exercise and by quantifying the three parameters of the adaptive response; namely, the time delay from onset of stimulus to onset of response, the time constant or rate of adaptation of the response, and the gain or amplitude of the response, we can better understand its control. Thus, in order to explore the accuracy of VOP as a technique to assess the hyperaemic response of the lower leg, we compared the parameters of these discrete phases with those obtained using DU.

Up until the work of Reeder and Green (Reeder and Green 2012), it was assumed that the dynamic response of blood flow during rest-exercise transitions only consisted of growth phases (Shoemaker et al. 1996a; Radegran and Saltin 1998; Hughson and Tschakovsky 1999; Saunders et al. 2005). However, Reeder and Green (Reeder and Green 2012) showed in healthy participants that this hyperaemic response to exercise is more dynamic than previously thought and consists, in addition to growth phases, of an early rapid decay phase and, depending upon the intensity, a later and slower decay phase; patterns that were also recently observed in individuals with type 2 diabetes (Kiely et al. 2014). The tri- and quadraphasic responses, including growth and decay phases, reported in the current study and obtained using both DU and VOP techniques, are therefore in agreement with this notion. In the current study, as in the study of Reeder and Green (Reeder and Green 2012) short duty cycles (1 s contraction / $2 \mathrm{~s}$ relaxation) allowed for better empirical modelling to determine these phases. It is possible that the longer duty cycles used in previous research (Shoemaker et al. 1996a; Radegran and Saltin 1998; Hughson and Tschakovsky 1999; Saunders et al. 2005) may help explain why growth-only LBF responses were observed. As previously reported by Reeder and Green (Reeder and Green 2012), in the present study evidence for the biphasic decay in blood flow was more apparent at 30 and 50\% MVC intensities than at the 70\% MVC intensity, which may be due to a higher increase in MAP over the latter part of exercise as was the case in the present study (data not shown). This is most likely as a result of an increase in vascular resistance in inactive regions due to an increase in sympathetic nervous activity. Further possibilities may relate to activation of antagonist muscles or the increased variability of blood flow responses at higher intensities.

In the present study, despite some relatively small differences in amplitudes of LBF responses (see immediately below), importantly, the time constant and time delay of all phases of the hyperaemic response at the 3 different intensities were similar between DU and VOP techniques. This suggests that VOP and DU provide similar trends on estimates of the dynamic response of blood flow during plantar-flexion exercise at intensities between 30 and $70 \%$ MVC. 


\section{Amplitudes of $L B F$ at rest and during exercise}

Prior to this study, Green et al. (2011) compared VOP and DU estimates of leg blood flow during relaxation periods between intermittent calf contractions at a wide range of intensities ( 50 to $400 \mathrm{~N}$ ) and observed a strong association (Pearson $r=0.91)$ between all paired responses reporting similar LBF values between techniques (248 and 278 $\mathrm{ml} / \mathrm{min}$ for VOP and DU) (Green et al. 2011). In that study ordinary least products (OLP) regression revealed a slope of 0.942 indicating a proportional bias of $\sim 6 \%$ between measurements techniques, VOP showing lower estimates of the hyperaemic response. In addition, OLP regression revealed a y-intercept of $-13 \mathrm{ml} / \mathrm{min}$ indicating a fixed bias between measurements and that VOP estimates were consistently lower by $\sim 13 \mathrm{ml} / \mathrm{min}$, a difference in LBF that was most likely established at rest (resting DU: $89 \mathrm{ml} / \mathrm{min}$ vs. resting VOP: $65 \mathrm{ml} / \mathrm{min} P<0.05$ ). Green and colleagues suggested that these fixed and proportional biases could be attributed to the fact that DU measures blood flow into vascular regions in the leg and foot which are not assessed by VOP (for more details see (Green et al. 2011)). In particular, the proportional bias between measurement techniques was attributed to the dilation of these additional vascular regions during exercise (Boushel et al. 2000) leading to a larger estimate of the hyperaemic response using DU than VOP (Green et al. 2011).

In agreement with Green et al. (2011), in the present study resting LBF values were significantly lower using VOP compared with DU (72 vs. $172 \mathrm{ml} / \mathrm{min}$ ); however, the magnitude of the difference was greater in the present study than in the study by Green and co-workers (Green et al. 2011). Resting LBF values using VOP reported in the present study are similar to those observed by previous studies in healthy male participants (Kiely et al. 2014; Green et al. 2011). However, previous observations of resting leg (i.e. popliteal) blood flow values using DU in healthy males are much more variable, as values of 45-50 ml/min (Villar and Hughson 2013), $89 \mathrm{ml} / \mathrm{min}$ (Green et al. 2011), $240 \mathrm{ml} / \mathrm{min}$ (Hunt et al. 2013) or up to $272 \mathrm{ml} / \mathrm{min}$ (Hunt et al. 2016) have been reported in the recent literature. This would suggest that in addition to the differences in the vascular regions assessed by both techniques, errors associated with both techniques (perhaps more so to DU, see limitations below) have influenced the values at rest.

Regarding LBF responses during exercise, in the present study hyperaemic responses at intensities equivalent to $30 \%$ and $50 \%$ MVC were $\sim 6 \%$ larger for DU compared with VOP. These findings are consistent with a similar proportional bias ( $\sim 6 \%$ ) shown by Green et al. (2011) during calf plantar-flexion exercise at a range of intensities 
up to $\sim 50 \%$ MVC (Green et al. 2011). At the intensity of $70 \%$ MVC in the present study the magnitude of the difference between techniques was larger $(\sim 16 \%$ larger for DU than VOP) with end-amplitudes at this higher intensity being significantly lower for VOP compared with DU (Table 1, Fig 4 D-F). It is possible that further dilation of the above-mentioned additional vascular regions which are measured by DU but not by VOP and that have been suggested to contribute to the $6 \%$ bias up to the intensities of $50 \%$ MVC might contribute to this larger proportional bias of $16 \%$ at the higher intensities of $70 \%$ MVC. The significantly larger overall popliteal artery diameters observed at 70\% MVC compared with the 2 lower intensities in the present study supports that further dilation of additional active vascular regions at this highest intensity is likely, although the increase in diameter might also be due to increased blood pressure in the popliteal artery. An alternative reason for this larger proportional bias between techniques at $70 \% \mathrm{MVC}$ is the build up of venous volume, and therefore, venous pressure, which can result in a reduction of arterial inflow (Tschakovsky et al. 1995) and/or a rise to leakage with the mechanical effect of muscle contraction (Lorentsen et al. 1970; Pallares et al. 1994; Egaña and Green 2005), possibly through the deep femoral vein if the cuff pressure becomes too low to collapse deep veins. Both conditions would further underestimate VOP measurements more than DU measurements. However, in the present study we did not observe evidence of venous outflow (i.e. presence of a plateau or reduction in the pulsatile change in leg volume between contractions) in the VOP recordings at any of the intensities. Even if the amplitudes of the first growth phase $\left(\mathrm{A}_{1}\right)$ were not significantly different between techniques they tended to be larger for VOP (Table 1, Fig 4 A-C). The reasons for this difference between techniques is not known, but one of the possible reasons, particularly at the lowest workload, relates to an early redistribution of the flow from leg/foot regions to calf muscles which would be detected by VOP but not by DU. The fact that End A estimates detected by VOP were not larger than estimates detected by DU is consistent with this and suggests that this redistributed flow is similar to the additional flow measured at rest by DU.

\section{Limitations}

VOP cannot assess blood flow during contraction and this represents a limitation. Another limitation relating to VOP is the fact that the use of the venous occlusion cuff could affect LBF responses during exercise (Radegran 1999; Tschakovsky et al. 1995). Tschakovsky et al. (1995) observed a significant decrease in the blood flow response (measured using DU) from the second to the fourth cardiac cycle when a venous occlusion pressure of 50 mmHg was used compared with no cuff pressure following a sustained contraction of the forearm; however, the initial cardiac cycle was unaffected by the cuff pressure (Tschakovsky et al. 1995). Given that in the present study 
each relaxation period included 1 or, at the most, 2 cardiac cycles, it is likely that the use of cuff inflation did not restrict estimates of LBF (or restrictions were very small). Furthermore, Green et al. (2011) compared intermittent plantar-flexion contractions at intensities between 200 to $600 \mathrm{~N}$ with and without cuff inflation (to $55 \mathrm{mmHg}$ ) using 1s contraction $-2 \mathrm{~s}$ relaxation duty cycles, and demonstrated that cuff inflation did not decrease LBF responses (Green et al. 2011).

Using DU, systematic errors in the measurement of arterial diameter, insonation angle and sample volume can cause large errors in measurement of blood flow during exercise, although the extent of these errors and the subsequent impact on the LBF measurements has not been quantified During low-intensity (8-13\% MVC) intermittent exercise muscle contraction has been shown to induce variability in the angle of insonation of $\sim 5 \%$ (Shoemaker et al. 1996b), where an error of $3^{\circ}$ when the angle of insonation was $55^{\circ}$ would overestimate femoral and axillary blood velocities by $8 \%$ (Amundsen et al. 2002). This variability in the angle of insonation was likely to be larger in the current study given that higher intensities were investigated and the popliteal artery was employed.

\section{Perspective}

In peripheral vascular disease metabolic control and fatigue resistance during exercise are impaired, and these effects are manifested at the onset of contracting calf muscle exercise (Barker et al. 2001; Barker et al. 2004; Bauer et al. 1999). Given that metabolic control and fatigue resistance during exercise are closely linked with the hyperaemic response in active muscles (Hughson et al. 2001), it is critically important to adequately measure the hyperaemic response in these altered muscle responses in order to elucidate the role of the hyperaemic response in these effects (Brass et al. 2004). In limbs with vascular disease complications induced by a collateralized arterial supply, arterial calcification and/or 'jetting' effects at the site of DU insonation make the DU technique, a criterion non-invasive technique for such assessment in healthy limbs, not well suited for these measurements in individuals with vascular disease; while VOP would not be influenced by these complications. In addition, in diseases where arteries can be affected by arteriosclerosis (e.g., coronary heart disease, diabetes), atherosclerotic changes to major arteries increase the challenge of assessing luminal diameter and blood flow with DU, whereas no adjustments are needed when using the VOP technique and thus seems to be better suited than DU to the assessment of the hyperaemic response in limbs with sclerotic arteries.

\section{Conclusions}


The current study showed similar trends on estimates of dynamic responses of muscle blood flow during calf plantar-flexion exercise at intensities equivalent to $30 \%, 50 \%$ and $70 \%$ MVC between VOP and DU measurement techniques considering slight differences in vascular regions assessed by these techniques and the likely sources of errors in both of them. These data suggest that VOP could be used as an alternative technique to DU for the assessment of LBF kinetics in healthy limbs. Further studies are needed to elucidate if VOP is better suited than DU to assess exercise hyperaemia in limbs with vascular disease.

\section{Acknowledgements}

The authors thank Mr David Fletcher (Trinity College Dublin) for his valuable contribution re-designing the calf ergometer.

\section{Grants}

This publication has emanated from research conducted with the financial support of Health Research Board (Grant No HRA_POR/2073/274)

\section{Compliance with ethical standards}

Conflict of interest Authors declare that they have no conflict of interest.

\section{Figure legends}

Fig 1: Mean ( \pm SD) VOP and DU leg blood flow responses at 30\% (A), 50\% (B) and 70\% (C) MVC intensities during calf exercise. Blood flow responses were taken during the relaxation phases of the calf exercise.

Fig 2. LBF responses during calf exercise using VOP (left panels) and DU (right panels) in a representative individual. Responses predicted from the fitting of either triphasic function (two growth and one decay phases) or quadraphasic function (two growth and two decay phases) are also shown with solid grey lines. A, VOP at 30\% MVC; B, VOP at 50\% MVC; C, VOP at 70\% MVC; D, DU at 30\% MVC; E, DU at 50\% MVC and F, DU at $70 \%$ MVC). Blood flow responses were taken during the relaxation phases of the calf exercise. 
Fig 3. Mean ( \pm SD) DU popliteal blood velocity (PBV) and popliteal artery diameters (PDiam) at $30 \%, 50 \%$ and $70 \%$ MVC intensities during calf exercise. PBV and PDiam measurements were taken during the relaxation phases of the calf exercise.

Fig 4. Bland Altman plots comparing $A_{1}$ (left panels) and End A (right panels) leg blood flow responses between VOP and DU. A, $\mathrm{A}_{1}$ at $30 \% \mathrm{MVC} ; \mathrm{B}, \mathrm{A}_{1}$ at $50 \% \mathrm{MVC} ; \mathrm{C}, \mathrm{A}_{1}$ at $70 \% \mathrm{MVC}$; D, End $\mathrm{A}$ at $30 \% \mathrm{MVC}$; E, End $\mathrm{A}$ at $50 \% \mathrm{MVC}$ and $\mathrm{F}$, End A at $70 \%$ MVC. Difference $\mathrm{A}_{1}=\mathrm{A}_{1 \mathrm{DU}}-\mathrm{A}_{1 \mathrm{VOP}}$, Average $\mathrm{A}_{1}=\left(\mathrm{A}_{1 \mathrm{DU}}-\mathrm{A}_{1 \mathrm{VOP}}\right) / 2 ;$ Difference End $\mathrm{A}=$ End $\mathrm{A}_{\mathrm{DU}}-$ End $\mathrm{A}_{\mathrm{VOP}}$, Average End $\mathrm{A}=\left(\right.$ End $\mathrm{A}_{\mathrm{DU}}-$ End $\left.\mathrm{A}_{\mathrm{VOP}}\right) / 2$. Solid horizontal lines show mean values and dashed horizontal lines represent $95 \%$ confidence limits.

\section{References}

Adiseshiah M, Barber RW, Szaz KF (1984) Measurement of regional lower limb blood flow in normal humans by inhalation of 133Xe. Eur J of Nucl Med 9(8):379-381

Amundsen BH, Wisloff U, Helgerud J, Hoff J, Slordahl SA (2002) Ultrasound recorded axillary artery blood flow during elbow-flexion exercise. Med Sci Sports Exerc 34(8):1288-1293

Andersen P, Saltin B (1985) Maximal perfusion of skeletal muscle in man. J Physiol 366(233-249

Anderson JD, Epstein FH, Meyer CH, Hagspiel KD, Wang H, Berr SS, Harthun NL, Weltman A, Dimaria JM, West AM, Kramer CM (2009) Multifactorial determinants of functional capacity in peripheral arterial disease: uncoupling of calf muscle perfusion and metabolism. J Am Coll Cardiol 54(7):628-635

Barcroft H, Millen JL (1939) The blood flow through muscle during sustained contraction. J Physiol 97(1):17-31

Barker GA, Green S, Askew CD, Green AA, Walker PJ (2001) Effect of propionyl-L-carnitine on exercise performance in peripheral arterial disease. Med Sci Sports Exerc 33(9):1415-1422

Barker GA, Green S, Green AA, Walker PJ (2004) Walking performance, oxygen uptake kinetics and resting muscle pyruvate dehydrogenase complex activity in peripheral arterial disease. Clin Sci (Lond) $106(3): 241-249$

Bauer TA, Regensteiner JG, Brass EP, Hiatt WR (1999) Oxygen uptake kinetics during exercise are slowed in patients with peripheral arterial disease. J Appl Physiol 87(2):809-816

Bernink PJ, Lubbers J, Barendsen GJ, van den Berg J (1982) Blood flow in the calf during and after exercise: measurements with Doppler ultrasound and venous occlusion plethysmography in healthy subjects and in patients with arterial occlusive disease. Angiology 33(3):146-160 
Boushel R, Langberg H, Green S, Skovgaard D, Bulow J, Kjaer M (2000) Blood flow and oxygenation in peritendinous tissue and calf muscle during dynamic exercise in humans. J Physiol 524 Pt 1(305-313

Brass EP, Hiatt WR, Green S (2004) Skeletal muscle metabolic changes in peripheral arterial disease contribute to exercise intolerance: a point-counterpoint discussion. Vasc Med 9(4):293-301

Bystrom S, Jensen B, Jensen-Urstad M, Lindblad LE, Kilbom A (1998) Ultrasound-Doppler technique for monitoring blood flow in the brachial artery compared with occlusion plethysmography of the forearm. Scand J Clin Lab Invest 58(7):569-576

duManoir GR, DeLorey DS, Kowalchuk JM, Paterson DH (2010) Kinetics of VO2 limb blood flow and regional muscle deoxygenation in young adults during moderate intensity, knee-extension exercise. Eur J Appl Physiol 108(3):607-617

Egaña M, Green S (2005) Effect of body tilt on calf muscle performance and blood flow in humans. J Appl Physiol 98(6):2249-2258

Egaña M, Green S (2007) Intensity-dependent effect of body tilt angle on calf muscle fatigue in humans. Eur J Appl Physiol 99(1):1-9

Gonzalez-Alonso J, Mortensen SP, Dawson EA, Secher NH, Damsgaard R (2006) Erythrocytes and the regulation of human skeletal muscle blood flow and oxygen delivery: role of erythrocyte count and oxygenation state of haemoglobin. J Physiol 572(Pt 1):295-305

Green S (2002) Haemodynamic limitations and exercise performance in peripheral arterial disease. Clin Physiol Funct Imaging 22(2):81-91

Green S, Thorp R, Reeder EJ, Donnelly J, Fordy G (2011) Venous occlusion plethysmography versus Doppler ultrasound in the assessment of leg blood flow during calf exercise. Eur J Appl Physiol 111(8):18891900

Heinonen I, Nesterov SV, Kemppainen J, Nuutila P, Knuuti J, Laitio R, Kjaer M, Boushel R, Kalliokoski KK (2007) Role of adenosine in regulating the heterogeneity of skeletal muscle blood flow during exercise in humans. J Appl Physiol 103(6):2042-2048

Hiatt WR, Huang SY, Regensteiner JG, Micco AJ, Ishimoto G, Manco-Johnson M, Drose J, Reeves JT (1989) Venous occlusion plethysmography reduces arterial diameter and flow velocity. J Appl Physiol $66(5): 2239-2244$

Hughson RL, Tschakovsky ME (1999) Cardiovascular dynamics at the onset of exercise. Med Sci Sports Exerc 31(7):1005-1010 
Hughson RL, Tschakovsky ME, Houston ME (2001) Regulation of oxygen consumption at the onset of exercise. Exerc Sport Sci Rev 29(3):129-133

Hunt JE, Galea D, Tufft G, Bunce D, Ferguson RA (2013) Time course of regional vascular adaptations to low load resistance training with blood flow restriction. J Appl Physiol 115(3):403-411

Hunt JE, Stodart C, Ferguson RA (2016) The influence of participant characteristics on the relationship between cuff pressure and level of blood flow restriction. Eur J Appl Physiol 116(7):1421-1432

Jorfeldt L, Rutberg H (1990) Comparison of dye-dilution and plethysmographic blood flow measurements: an evaluation of the influence of invasive techniques on blood flow and on arterial and femoral venous substrate variables in man. Clin Sci (Lond) 79(1):81-87

Joyner MJ, Dietz NM, Shepherd JT (2001) From Belfast to Mayo and beyond: the use and future of plethysmography to study blood flow in human limbs. J Appl Physiol 91(6):2431-2441

Kaulesar Sukul DM, den Hoed PT, Johannes EJ, van Dolder R, Benda E (1993) Direct and indirect methods for the quantification of leg volume: comparison between water displacement volumetry, the disk model method and the frustum sign model method, using the correlation coefficient and the limits of agreement. Journal of biomedical engineering 15(6):477-480

Kiely C, O'Connor E, O'Shea D, Green S, Egaña M (2014) Hemodynamic responses during graded and constantload plantar flexion exercise in middle-aged men and women with type 2 diabetes. J Appl Physiol $117(7): 755-764$

Koskolou MD, Calbet JA, Radegran G, Roach RC (1997) Hypoxia and the cardiovascular response to dynamic knee-extensor exercise. Am J Physiol 272(6 Pt 2):H2655-2663

Lorentsen E, Hansteen V, Sivertssen E (1970) The influence of venous collecting pressure on measurements of calf blood flow by venous occlusion plethysmography. Angiology 21(10):661-677

Lubbers J, Bernink PJ, Barendsen GJ, van den Berg JW (1979) A continuous wave Doppler velocimeter for monitoring blood flow in the popliteal artery, compared with venous occlusion plethysmography of the calf. Pflugers Arch 382(3):241-248

MacAnaney O, Reilly H, O'Shea D, Egaña M, Green S (2011) Effect of type 2 diabetes on the dynamic response characteristics of leg vascular conductance during exercise. Diab Vasc Dis Res 8(1):12-21

MacDonald MJ, Shoemaker JK, Tschakovsky ME, Hughson RL (1998) Alveolar oxygen uptake and femoral artery blood flow dynamics in upright and supine leg exercise in humans. J Appl Physiol 85(5):16221628 
Pallares LC, Deane CR, Baudouin SV, Evans TW (1994) Strain gauge plethysmography and Doppler ultrasound in the measurement of limb blood flow. Eur J Clin Invest 24(4):279-286

Paterson DH, Jones GR, Rice CL (2007) Ageing and physical activity: evidence to develop exercise recommendations for older adults. Can J Public Health 98 Suppl 2(S69-108

Radegran G (1997) Ultrasound Doppler estimates of femoral artery blood flow during dynamic knee extensor exercise in humans. J Appl Physiol 83(4):1383-1388

Radegran G (1999) Limb and skeletal muscle blood flow measurements at rest and during exercise in human subjects. Proc Nutr Soc 58(4):887-898

Radegran G, Saltin B (1998) Muscle blood flow at onset of dynamic exercise in humans. Am J Physiol 274(1 Pt 2):H314-322

Ray CA, Rea RF, Clary MP, Mark AL (1993) Muscle sympathetic nerve responses to dynamic one-legged exercise: effect of body posture. Am J Physiol 264(1 Pt 2):H1-7

Reeder EJ, Green S (2012) Dynamic response characteristics of hyperaemia in the human calf muscle: effect of exercise intensity and relation to electromyographic activity. Eur J Appl Physiol 112(12):3997-4013

Saltin B (2007) Exercise hyperaemia: magnitude and aspects on regulation in humans. J Physiol 583(Pt 3):819823

Saunders NR, Pyke KE, Tschakovsky ME (2005) Dynamic response characteristics of local muscle blood flow regulatory mechanisms in human forearm exercise. J Appl Physiol 98(4):1286-1296

Shoemaker JK, Phillips SM, Green HJ, Hughson RL (1996a) Faster femoral artery blood velocity kinetics at the onset of exercise following short-term training. Cardiovasc Res 31(2):278-286

Shoemaker JK, Pozeg ZI, Hughson RL (1996b) Forearm blood flow by Doppler ultrasound during test and exercise: tests of day-to-day repeatability. Med Sci Sports Exerc 28(9):1144-1149

Tschakovsky ME, Shoemaker JK, Hughson RL (1995) Beat-by-beat forearm blood flow with Doppler ultrasound and strain-gauge plethysmography. J Appl Physiol 79(3):713-719

Villar R, Hughson RL (2013) Effect of altered arterial perfusion pressure on vascular conductance and muscle blood flow dynamic response during exercise in humans. J Appl Physiol 114(5):620-627 
Table 1: Dynamic response characteristics of the first three phases of leg blood flow obtained with VOP and DU during calf plantar-flexion at 30\%, 50\% and 70\% MVC exercise intensities.

\begin{tabular}{|c|c|c|c|c|c|c|}
\hline & \multicolumn{2}{|c|}{$30 \% \operatorname{MVC}(\mathrm{n}=10)$} & \multicolumn{2}{|c|}{$50 \% \operatorname{MVC}(\mathrm{n}=10)$} & \multicolumn{2}{|c|}{$70 \% \operatorname{MVC}(\mathrm{n}=10)$} \\
\hline & VOP & DU & VOP & DU & VOP & $\mathrm{DU}$ \\
\hline $\mathrm{A}_{0}(\mathrm{ml} / \mathrm{min})$ & $71 \pm 33^{*}$ & $168 \pm 48$ & $66 \pm 35^{*}$ & $171 \pm 49$ & $73 \pm 35^{*}$ & $173 \pm 47$ \\
\hline $\mathrm{A}_{1}(\mathrm{ml} / \mathrm{min})$ & $301 \pm 132$ & $210 \pm 74$ & $448 \pm 131$ & $372 \pm 150$ & $677 \pm 177$ & $656 \pm 291$ \\
\hline $\mathrm{TD}_{1}(\mathrm{~s})$ & $0.5 \pm 0.3$ & $0.6 \pm 0.3$ & $0.6 \pm 0.1$ & $0.7 \pm 0.2$ & $0.6 \pm 0.2$ & $0.6 \pm 0.2$ \\
\hline$\tau_{1}(\mathrm{~s})$ & $3.0 \pm 2.6$ & $2.6 \pm 1.4$ & $2.6 \pm 1.2$ & $2.7 \pm 1.4$ & $3.9 \pm 1.6$ & $3.5 \pm 1.1$ \\
\hline $\mathrm{A}_{2}$ (decay) $(\mathrm{ml} / \mathrm{min})$ & $116 \pm 52$ & $120 \pm 90$ & $165 \pm 50$ & $169 \pm 95$ & $265 \pm 78$ & $258 \pm 130$ \\
\hline $\mathrm{TD}_{2}$ (decay) (s) & $11.9 \pm 7.4$ & $12.7 \pm 4.6$ & $10.7 \pm 3.2$ & $10.5 \pm 3.5$ & $12.3 \pm 3.4$ & $11.2 \pm 2.5$ \\
\hline$\tau_{2}($ decay) $(\mathrm{s})$ & $7.3 \pm 7.4$ & $5.7 \pm 2.9$ & $5.7 \pm 4.2$ & $6.5 \pm 4.9$ & $5.3 \pm 2.7$ & $3.3 \pm 2.0$ \\
\hline $\mathrm{A}_{3}(\mathrm{ml} / \mathrm{min})$ & $98 \pm 46$ & $103 \pm 82$ & $185 \pm 59$ & $214 \pm 78$ & $181 \pm 67$ & $227 \pm 91$ \\
\hline $\mathrm{TD}_{3}(\mathrm{~s})$ & $24.7 \pm 9.5$ & $22.9 \pm 7.3$ & $22.5 \pm 5.6$ & $22.6 \pm 6.1$ & $21.4 \pm 4$ & $20.1 \pm 4.5$ \\
\hline$\tau_{3}(\mathrm{~s})$ & $20.4 \pm 10.5$ & $27.2 \pm 19.7$ & $19.0 \pm 11.9$ & $16.1 \pm 7.3$ & $9.1 \pm 4.9$ & $11.0 \pm 3.9$ \\
\hline End A (ml/min) & $295 \pm 92$ & $307 \pm 75$ & $476 \pm 159$ & $512 \pm 137$ & $621 \pm 217^{*}$ & $785 \pm 260$ \\
\hline
\end{tabular}

Data are means $\pm \mathrm{SD} . \mathrm{A}_{0}$ baseline amplitude; $\mathrm{A}_{1}, \mathrm{~A}_{2}$ and $\mathrm{A}_{3}$, amplitudes, $\mathrm{TD}_{1}, \mathrm{TD}_{2}$ and $\mathrm{TD}_{3}$, time delays and $\tau_{1}$, $\tau_{2}$ and $\tau_{3}$, time constants of the first (rapid growth), second (rapid decay) and third (slow growth) phases respectively.

* significantly different than DU within same exercise intensity $(P<0.05)$ 
Figure 1

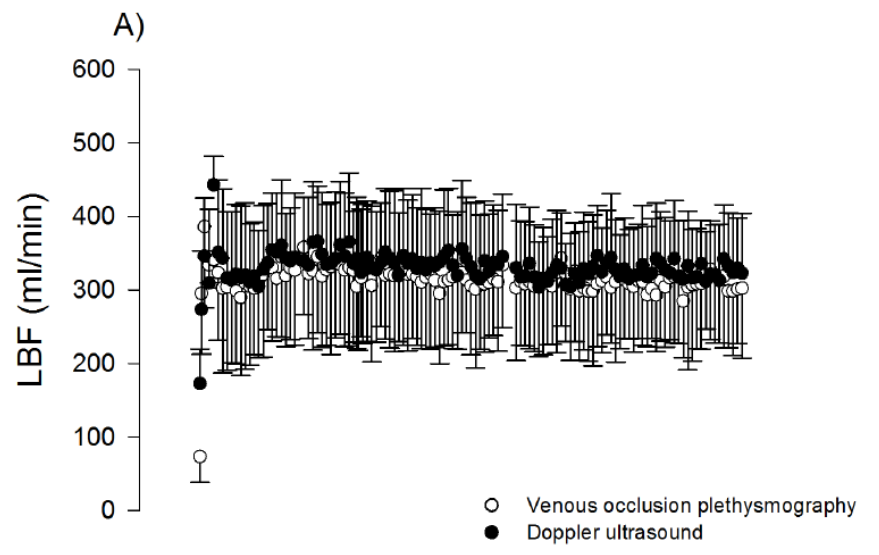

B)

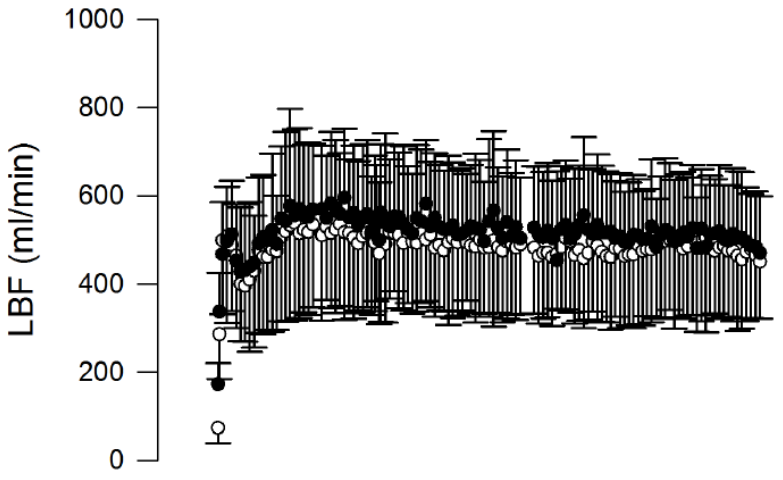

C)

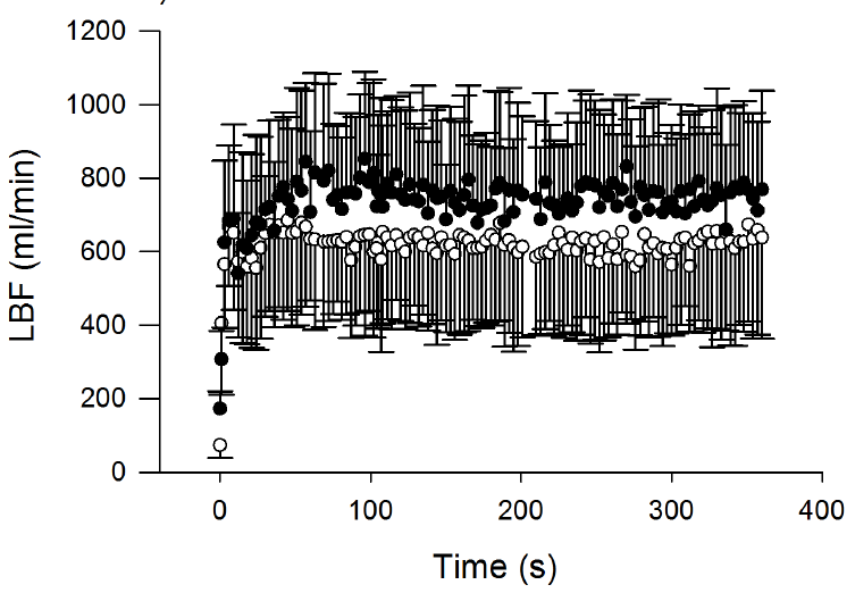


Figure 2

A)

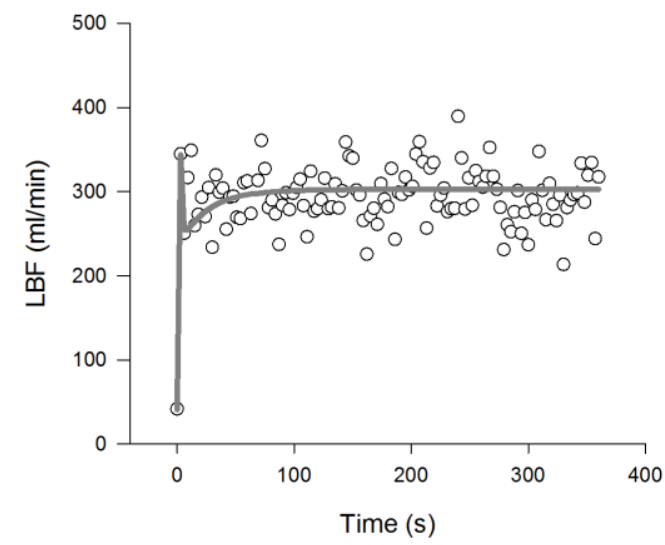

B)

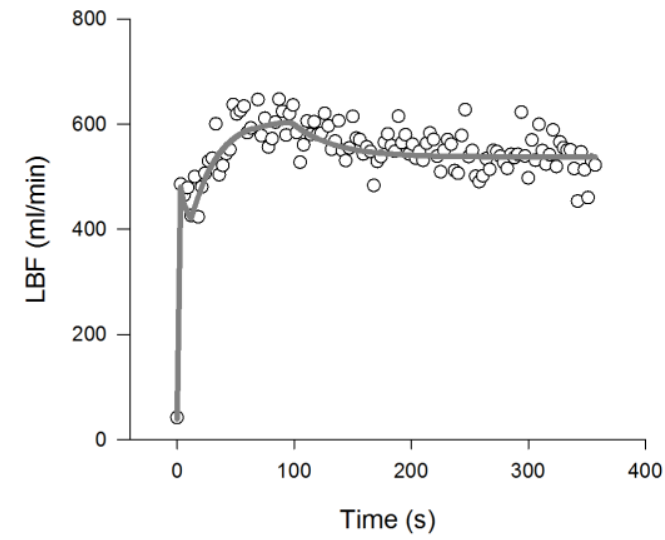

C)

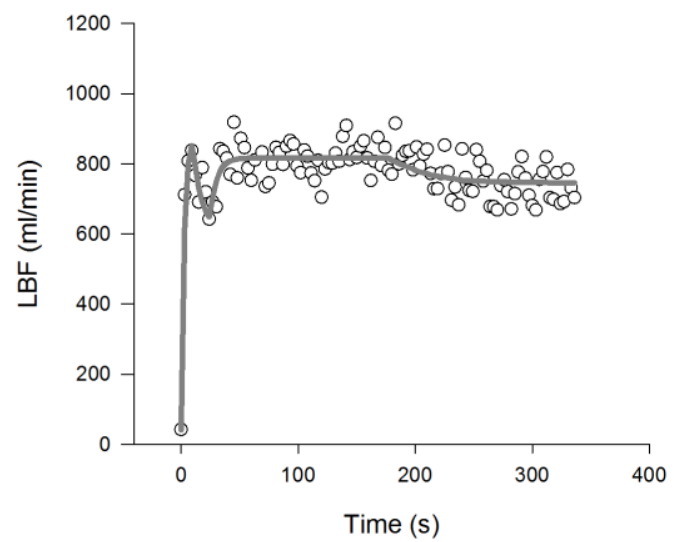

D)

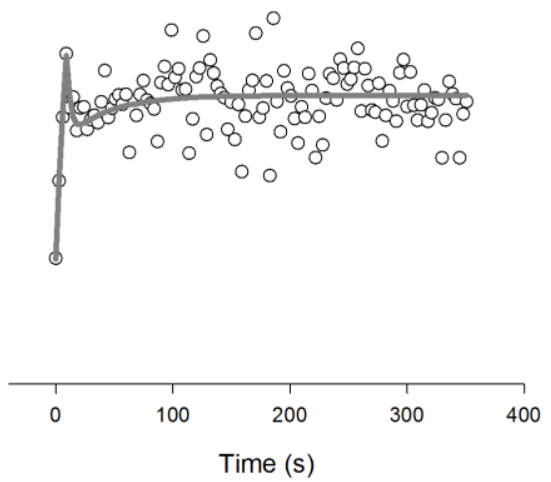

E)

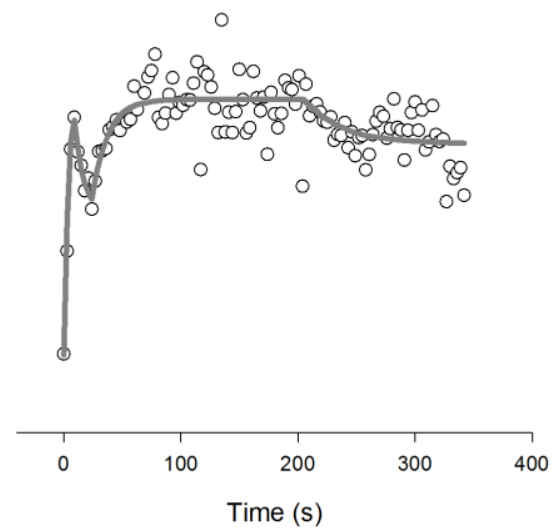

F)

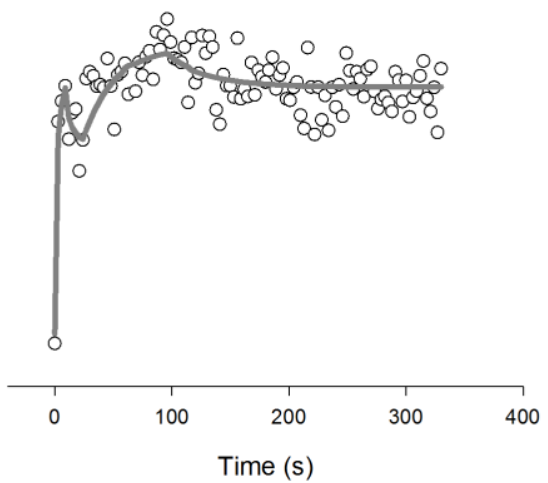


Figure 3

$30 \%$ MVC
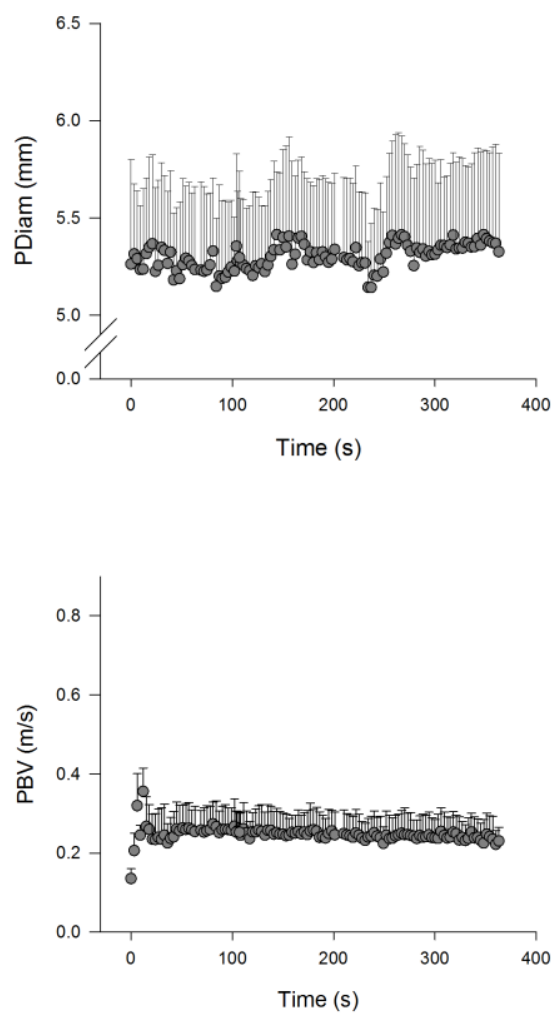

$50 \%$ MVC
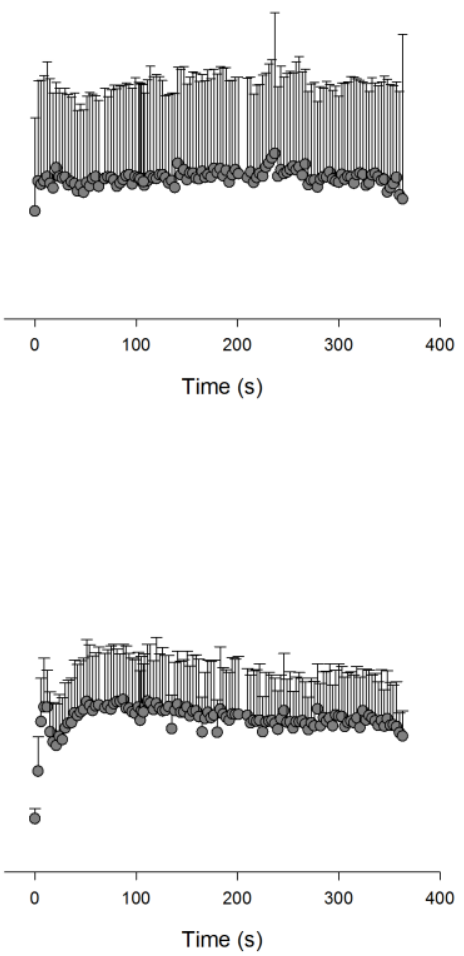

$70 \%$ MVC
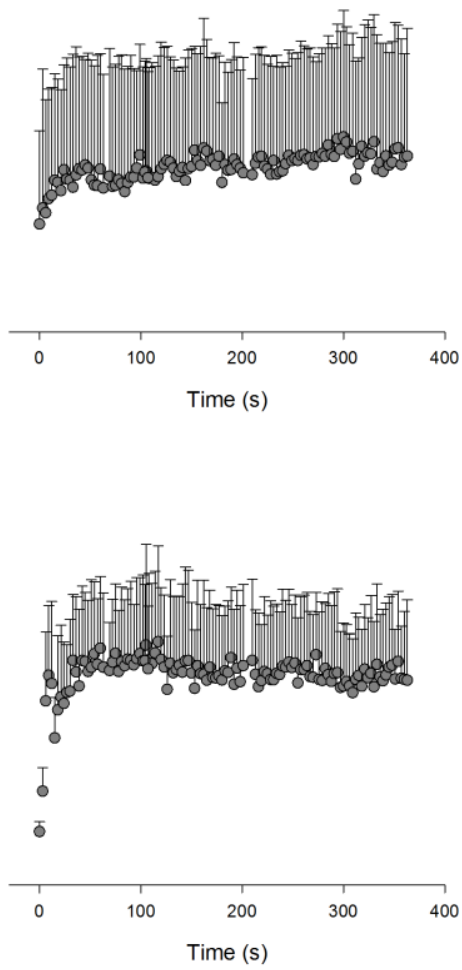
Figure 4

A)

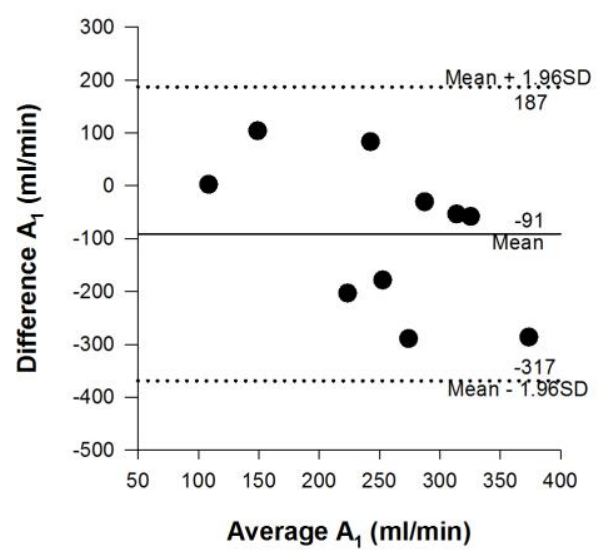

B)

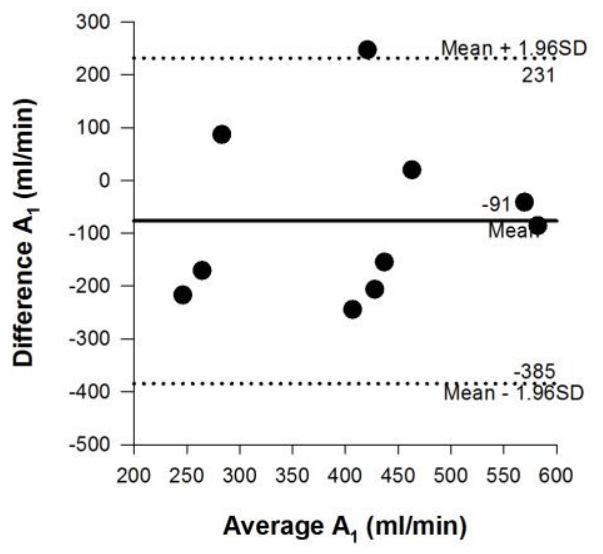

C)

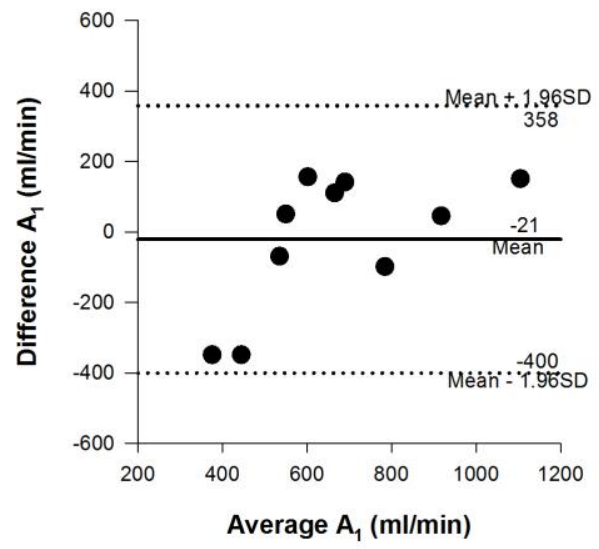

D)

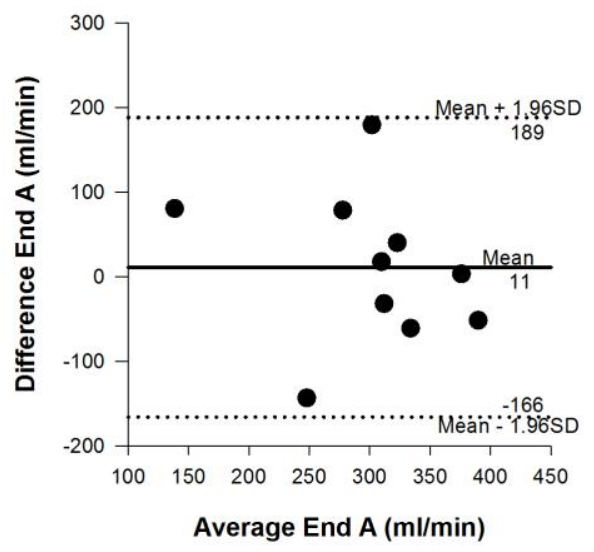

E)

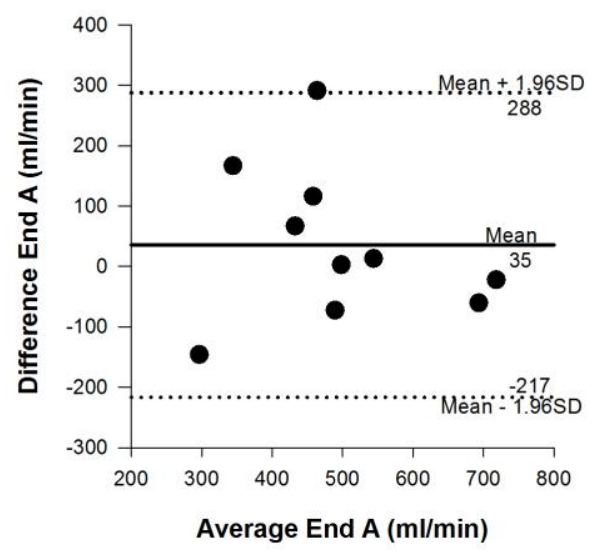

F)

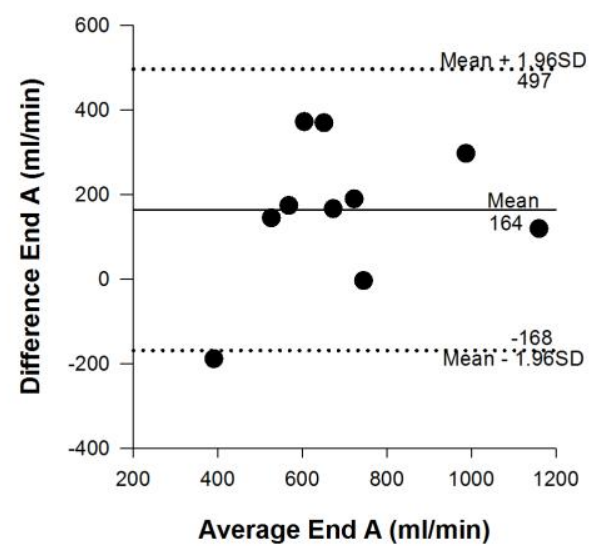

